\title{
EDITORIAL
}

\section{The race for a coronavirus vaccine}

Gerardo Ferbeyre ${ }^{1}$, Nelson Santiago Vispo ${ }^{2}$

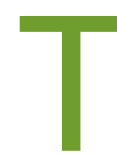

he international race to find a preventive vaccine and effective treatments against COVID 19 has been influenced by two fundamental factors. Firstly, by the molecular characterization of the causative virus and the pathology it produces, and secondly, by access to this information, mostly free of charge by the international scientific community causing a synergy to obtain results in such a short time.

Several vaccines preparations against Covid19 have entered Phase 3 clinical trials. Although it is uncertain the degree of protection that they will achieve, preliminary data from Phase 1 and 2 trials and studies in animals indicate that they trigger an antiviral immune response without serious side effects. The current formulations include viral vectors, RNA vaccines, inactivated viruses, and recombinant proteins particles. They all have advantages and disadvantages, but only the results of Phase 3 clinical trials will ultimately decide the best candidates for vaccination campaigns.

The tremendous impact of the SARS-CoV-2 in our society has triggered an unprecedented effort to find a vaccine to control the pandemic. Billions of dollars have already been invested in multiple vaccination schemes. According to the WHO, more than 170 vaccines were in different phases of development in August 2020. Here is a summary of the advantages and disadvantages of the front runner strategies categorized according to their delivery method (Figure 1).
DOI. 10.21931/RB/2020.05.04.1

\section{Viral Vectors}

Several vaccines against SARS-CoV-2 take advantage of viral vectors to express the viral proteins in the host. These vectors mimic a natural viral infection leading to the presentation of viral antigens to the immune system. Vectors based on human adenovirus were used before to make a vaccine against Ebola with excellent results and were the first to be used to make a vaccine against Coronavirus. The main disadvantage of these vectors is the presence of antibodies against adenovirus in the human population ${ }^{1}$. Also, after the first shot, patients will make antibodies against the viral vector making it very difficult to make a booster, second shot. The most widely used adenovirus vector comes from the strain Ad5. The CanSino/China vaccine is based on Ad5 expressing the full-length spike gene? One potential problem with Ad5 is that it is a widespread strain and most people have antibodies against it ${ }^{1}$. To circumvent this problem, the Gamaleya Research Institute in Russia designed a vaccine based on two adenoviruses. The first is Ad26, which is relatively rare, and the second is Ad5. The use of two vectors facilitates the administration of the booster shot, but still, the use of Ad5 may reduce its efficacy. This vaccine, called Sputnik $V$, also showed immunogenicity and short term safety in phase I/II trials published recently in the Lancet online. The University of Oxford partnered with AstraZeneca to make a vaccine using an adenovirus from chimpanzees, which is not recognized by

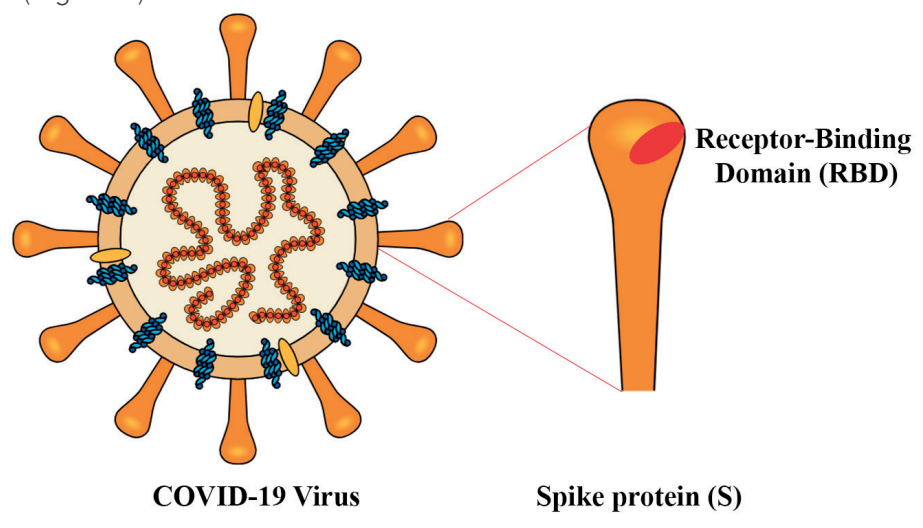

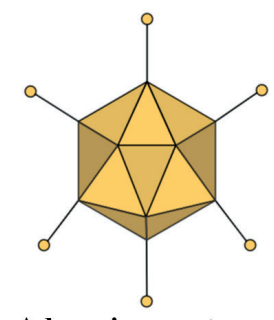

Adenovirus vector

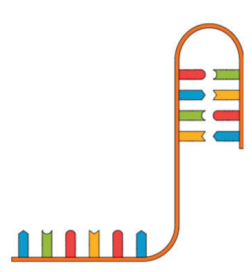

RNA Vaccines

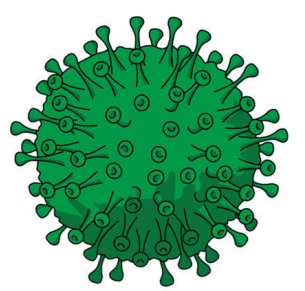

Inactivated Virus Vaccines

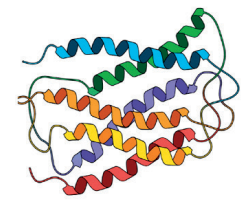

Recombinant protein

Figure 1. Different design strategies for new vaccine candidates based on nucleic acids, Adenovirus viral vectors, inactivated COVID virus or peptide sequences.

\footnotetext{
${ }^{1}$ Université de Montréal, Faculté de médicine, Département de biochimie et médecine moléculaire, Montréal, Canada.
}

${ }^{2}$ Universidad Yachay Tech, Imbabura, Ecuador. 
human anti-adenovirus antibodies. This was the first vaccine that reported successful safety and immunogenicity data back in July $2020^{3}$. The main disadvantage of this vaccine is that it is based on only one adenovirus. Antibodies developed against this vector will reduce the efficacy of a second shot.

\section{RNA Vaccines}

RNA vaccines have been experimentally tested in animals with success for many years but never approved for use in humans. These vaccines express antigens in host cells transiently mimicking a natural viral infection ${ }^{4,5}$. The Covid19 pandemic provided an opportunity for several companies to develop this innovative vaccination technology. Moderna tested their RNA vaccine mRNA-1273 encoding for the SARS-CoV-2 spike protein in a phase 1 trial documenting a good immunogenicity and safety profiles ${ }^{6}$. The German company BioNtech has partnered with Pfizer to perform Phase 3 clinical trials with their mRNA vaccine BNT162b2, which also encodes for the spike protein. They reported good immunogenicity both in young and older patients, and it is the first vaccine to demonstrate that they could protect the population at higher risk ${ }^{7}$. CureVac is also developing mRNA vaccines ${ }^{5}$, and they have one against Covid-19 in Phase I trials. One potential difficulty with RNA vaccines is that they require freezing at shallow temperatures to keep the RNA-lipid emulsion homogenous.

\section{Inactivated Virus Vaccines}

The anti-polio vaccine, widely used all over the world, is an inactivated virus ${ }^{8}$. This strategy was followed by SinoPharm in China, reporting a low rate of adverse effects and good immunogenicity with their SinoVac inactivated virus ${ }^{9}$. However, in a recent interview published in Science, Moncef Saloui, head of Operation Warp Speed, considered that this strategy is not a good idea ${ }^{10}$. Inactivated viruses may enhance the disease as reported for the inactivated respiratory syncytial virus vaccine. Also, there are safety concerns associated with producing high amounts of inactivated viruses. A single nucleotide change that arose during the preparation of the inactivated polio vaccine increased the neurovirulence of this vaccine ${ }^{8}$.

\section{Recombinant protein and Virus-Like Particles}

Recombinant proteins are poorly immunogenic, and when given alone, they require the use of adjuvants to elicit a long-lasting immune response ${ }^{11}$. The formation of virus-like particles (VLP) can increase the immunogenicity of recombinant proteins. VLP has been used before to create vaccines against HPV and Hepatitis B. The HPV vaccine is prepared from purified HPV L1 protein that self assembles to form the VLP capable of triggering a protective immune response ${ }^{12}$. The Hepatitis $B$ vaccine is prepared from recombinant $\mathrm{HBsAg}(\mathrm{He}-$ patitis B surface Antigen) produced in yeast ${ }^{13,14}$. The Quebec company Medicago has optimized the production of VLP from influenza haemagglutinin trimers in plants ${ }^{15}$, and they are now making Coronavirus Like particles using this technology to initiate Phase 2/3 trials in October. These particles need adjuvants that Medicago will add to their formulation using technologies from GSK and Dynavax. The company Novavax, reported a very successful phase 1-2 trial with the recombinant SARS-CoV-2 vaccine. Their vaccine is based on recombinant spike glycoproteins expressed in insect cells from a baculovirus vector with matrix $\mathrm{Ml}$, a saponin based adjuvant ${ }^{16}$. One advantage of this formulation is that it can be stored at 2-8oC.

On August 18, the Cuban Ministry of Public Health announced that the Finlay Vaccine Institute in Havana began a clinical trial of a vaccine for Covid-19. The vaccine, called Soberana 1 , contains a part of the spike protein, called RBD, along with the meningococcal outer membrane vesicle system and alumina; the basis of VA-MENGOC, BC meningococcal vacci$n e^{17}$. This institution has widely studied the vesicle of the outer membrane of the meningococcal bacteria for many years as an enhancer of the innate immune response, and in fact, it has been studied as one of the pre-existing Cuban drugs in the fight against COVID-19 since the beginning of the pandemic ${ }^{18}$.

\section{Concluding remarks}

Many more vaccine candidates are at the initial stages of the investigation, but we should know the real potential and challenges of making a coronavirus vaccine from the results we will get with these initial candidates. All phase II trials demonstrated the presence of neutralizing antibodies in the blood of vaccinated patients, but it is not known whether these antibodies correlate with protection. There have been several anecdotal reports that people can be reinfected with SARSCoV-2, but the frequency of these events is still unknown. If reinfections are common, we may need to re-vaccinate the population as long as the virus remains circulating. Therefore the use of viral vectors may be compromised for frequent administration. Only one vaccine (BNT162b2) has shown good immunogenicity in old patients that are at high risk of both acquiring and dying from Covid-19. However, this only means that BioNtech/Pfizer tested this population in their Phase 2 trials, and data from other vaccine candidates are not yet available. Although adenovirus-based vaccines are now among the front runners, we cannot discard mRNA vaccines that can be used without the risk of inactivation. For similar reasons, other vaccines now in Phase 1-2 trials should be considered in the future.

All vaccines tested in Phase 1-2 trials have shown so far to have a good safety profile. If the results of Phase 3 trials that have enrolled thousands of patients confirm this trend, there is no reason to refuse vaccination for fear or political reasons. Even the low-risk population should be vaccinated to avoid passing the virus to high-risk populations. Nevertheless, the final word on both efficacy and toxicity is up to Phase 3 clinical trials that should be completed at the end of the year. It is remarkable that the Covid19 pandemic has shortened the time to develop a vaccine, which traditionally has taken as long as ten years. This is due in part to previous knowledge of another coronavirus (SARS) that identified the spike protein as a target for vaccine development ${ }^{19,20}$. We are lucky that we have many candidate vaccines in the pipeline. It is hard to believe that they will all fail and will not confer any degree of protection. In fact, although many vaccine trials fail, once they make it to phase 3 trials, they have an $85 \%$ chance of success. The CDC reports that the flu vaccines are regularly under $50 \%$ effective, but the influenza virus is known to mutate every season. Although there is evidence of mutations in SARS-CoV-2, we still do not know the magnitude of this phenomenon and whether these mutants are actually resistant to the previous immunity. Covid-19 has put science and health care systems to the test. It is clear that a rapid response was only possible due to advances in molecular biology and medical sciences. Further investment in basic research is the only way to be ready for future challenges. 


\section{Bibliographic references}

1. Barouch, D. H. et al. International seroepidemiology of adenovirus serotypes 5, 26, 35, and 48 in pediatric and adult populations. Vaccine 29, 5203-5209 (2011).

2. Zhu, F. C. et al. Immunogenicity and safety of a recombinant adenovirus type-5-vectored COVID-19 vaccine in healthy adults aged 18 years or older: a randomised, double-blind, placebo-controlled, phase 2 trial. Lancet 396, 479-488 (2020).

3. Folegatti, P. M. et al. Safety and immunogenicity of the ChAdOxI nCoV-19 vaccine against SARS-CoV-2: a preliminary report of a phase 1/2, single-blind, randomised controlled trial. Lancet 396, 467-478 (2020).

4. Alameh, M.-G., Weissman, D. \& Pardi, N. Messenger RNA-Based Vaccines Against Infectious Diseases. in Current topics in microbiology and immunology (Curr Top Microbiol Immunol, 2020). doi:10.1007/82_2020_202

5. Thran, $M$. et al. mRNA mediates passive vaccination against infectious agents, toxins, and tumors. EMBO Mol. Med. 9, 14341447 (2017).

6. Jackson, L. A. et al. An mRNA Vaccine against SARS-CoV-2 Preliminary Report. N. Engl. J. Med. (2020) doi:10.1056/nejmoa2022483.

7. Walsh, E. E. et al. RNA-Based COVID-19 Vaccine BNT162b2 Selected for a Pivotal Efficacy Study. medRxiv 2020.08.17.20176651 (2020) doi:10.1101/2020.08.17.20176651.

8. Evans, D. M. A. et al. Increased neurovirulence associated with a single nucleotide change in a noncoding region of the Sabin type 3 poliovaccine genome. Nature 314, 548-550 (1985)

9. Xia, S. et al. Effect of an Inactivated Vaccine Against SARS-CoV-2 on Safety and Immunogenicity Outcomes. Jama 1-10 (2020) doi:10.1001/jama.2020.15543.

10. Cohen, J. Leader of U.S. vaccine push says he'll quit if politics trumps science. Science (80-. ). (2020) doi:10.1126/science. abe6380.
11. Pérez, O. et al. Human prophylactic vaccine adjuvants and their determinant role in new vaccine formulations. Brazilian Journal of Medical and Biological Research vol, 45 681-692 (2012).

12. Garbuglia, A. R., Lapa, D., Sias, C., Capobianchi, M. R. \& Del Porto, P. The Use of Both Therapeutic and Prophylactic Vaccines in the Therapy of Papillomavirus Disease. Front. Immunol. 11, 1-14 (2020).

13. Ho, J. K. T., Jeevan-Raj, B. \& Netter, H. J. Hepatitis B Virus (HBV) Subviral Particles as Protective Vaccines and Vaccine Platforms. Viruses 12, 1-26 (2020).

14. Hardy, E. et al. Large-scale production of recombinant hepatitis B surface antigen from Pichia pastoris. J. Biotechnol. 77, 157-167 (2000).

15. Phane Pillet, S. et al. Immunogenicity and safety of a quadrivalent plant-derived virus like particle influenza vaccine candidate-Two randomized Phase II clinical trials in 18 to 49 and 50 years old adults. (2019) doi:10.1371/journal.pone.0216533.

16. Keech, C. et al. First-in-Human Trial of a SARS CoV 2 Recombinant Spike Protein Nanoparticle Vaccine. medRxiv 2020.08.05.20168435 (2020) doi:10.1101/2020.08.05.20168435.

17. Corum, J. \& Zimmer, C. Coronavirus vaccine tracker - The New York Times. The New York Times 1-17 (2020).

18. Tahimi Arboleya. Cuban Soberana 01 vaccine | OnCubaNews English. https://oncubanews.com/ https://oncubanews.com/en/ cuba/cuban-soberana-01-vaccine/ (2020).

19. Walls, A. C. et al. Structure, Function, and Antigenicity of the SARS-CoV-2 Spike Glycoprotein. Cell 181, 281-292.e6 (2020).

20.Zamora-Ledezma, C.; C., D.F.C.; Medina, E.; Sinche, F.; Santiago Vispo, N.; Dahoumane, S.A.; Alexis, F. Biomedical Science to Tackle the COVID-19 Pandemic: Current Status and Future Perspectives. Molecules 2020, 25,4620 . 